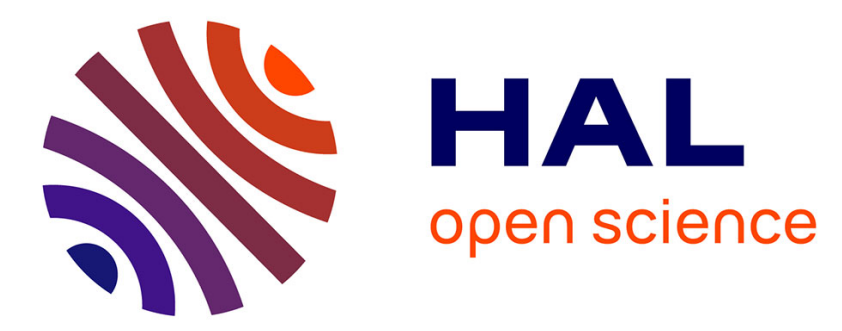

\title{
Etude de l'écoulement dans un collecteur plan
}

\author{
A.-M. Dalbert, J.-L. Peube, F. Penot, J.-F. Robert
}

\section{To cite this version:}

A.-M. Dalbert, J.-L. Peube, F. Penot, J.-F. Robert. Etude de l'écoulement dans un collecteur plan. Revue de Physique Appliquée, 1980, 15 (2), pp.201-206. 10.1051/rphysap:01980001502020100 jpa00244715

\section{HAL Id: jpa-00244715 https://hal.science/jpa-00244715}

Submitted on 1 Jan 1980

HAL is a multi-disciplinary open access archive for the deposit and dissemination of scientific research documents, whether they are published or not. The documents may come from teaching and research institutions in France or abroad, or from public or private research centers.
L'archive ouverte pluridisciplinaire HAL, est destinée au dépôt et à la diffusion de documents scientifiques de niveau recherche, publiés ou non, émanant des établissements d'enseignement et de recherche français ou étrangers, des laboratoires publics ou privés. 


\title{
Etude de l'écoulement dans un collecteur plan
}

\author{
A.-M. Dalbert, J.-L. Peube, F. Penot et J.-F. Robert \\ Laboratoire d'Energétique Solaire, 40, avenue du Recteur Pineau, 86022 Poitiers Cedex, France
}

(Reçu le 11 juillet 1979, révisé le 29 octobre 1979, accepté le 31 octobre 1979)

\begin{abstract}
Résumé. - On étudie les mouvements de convection mixte laminaire dans un collecteur plan vertical dans le cas où les parois sont soumises à des flux constants mais asymétriques. L'importance relative de la convection naturelle et de la convection forcée est discutée quand l'écoulement est pleinement développé. Dans la région de développement de l'écoulement, la résolution numérique des équations de la couche limite permet d'obtenir les profils de vitesse et de température dans le collecteur, en convection naturelle et mixte. On décrit ensuite une étude expérimentale dont les résultats sont discutés et comparés aux résultats numériques.
\end{abstract}

\begin{abstract}
Laminar mixed convection in a vertical flat plate collector has been investigated in the case of an asymmetric uniform wall heating. The relative importance of free and forced convection is discussed in the case of the fully developed flow. Numerical solutions are obtained in the case of the developing free and mixed convection flows. Tests have been carried out and numerical profiles and experimental measurements have been compared and discussed.
\end{abstract}

\begin{tabular}{|c|c|}
\hline Nomer & \\
\hline & Diffusivite thermique du fluide. \\
\hline & $\begin{array}{l}\text { Gradient de température moyenne } \\
\text { dimensionnel. }\end{array}$ \\
\hline & Demi-épaisseur du canal. \\
\hline & Accélération de la pesanteur. \\
\hline$r_{\mathrm{e}}, G, G r$ & $\begin{array}{l}\text { Nombres adimensionnels respectivement } \\
\text { définis par }(8),(25) \text { et }(28) \text {. }\end{array}$ \\
\hline & Hauteur du canal. \\
\hline & Nombre adimensionnel défini par (6). \\
\hline & r adimensionnelle du canal. \\
\hline & dimensionnel. \\
\hline $\begin{array}{l}p_{\mathrm{g}} \\
P,\end{array}$ & $\begin{array}{l}\text { Pression motrice, } \Delta p_{\mathrm{g}}=p_{\mathrm{g}}(H)-p_{\mathrm{g}}(0) \text {. } \\
\text { Pressions motrices adimensionnelles, } \\
D P_{\mathrm{g}}: \text { différence de pression motrice adi- } \\
\text { mensionnelle. }\end{array}$ \\
\hline$P, Q$ & $\begin{array}{l}\text { Nombres adimensionnels respectivement } \\
\text { définis par (7), (10) et (9). }\end{array}$ \\
\hline & $\begin{array}{l}\text { Densité de flux thermique sur la plaque } \\
\text { gauche. }\end{array}$ \\
\hline & $\begin{array}{l}\text { Densite de flux thermique sur la plaque } \\
\text { droite }\left(q_{2} \geqslant q_{1}\right) \text {. }\end{array}$ \\
\hline & Nombre adimensionnel défini par (22). \\
\hline & Température ambiante. \\
\hline & $\begin{array}{l}\text { Température transversale dimen } \\
\text { nelle. }\end{array}$ \\
\hline & $\begin{array}{l}\text { Température transversale adimen } \\
\text { nelle. }\end{array}$ \\
\hline
\end{tabular}

$T_{\mathrm{md}} \quad$ Température moyenne dimensionnelle.

$T_{\mathrm{m}} \quad$ Température moyenne adimensionnelle.

$u_{0} \quad$ Vitesse d'entrée du fluide.

$u, v \quad$ Vitesses dimensionnelles respectivement sur $x$ et $y$.

$U, U^{\prime}, V$ Vitesses adimensionnelles respectivement sur $x$ pour $U$ et $U^{\prime}$ et sur $y$ pour $V$.

$x, y \quad$ Coordonnées cartésiennes dimensionnelles.

$X$ ou $X^{\prime}, Y$ Coordonnées cartésiennes adimensionnelles.

$\beta \quad$ Coefficient de dilatation thermique du fluide.

Conductivité thermique du fluide.

Viscosité cinématique du fluide.

Masse volumique du fluide.

Température dimensionnelle.

Températures adimensionnelles.

\section{$\rho$ \\ $\theta_{\mathrm{d}}$ \\ $\theta, \theta^{\prime}$}

$\lambda$
1. Introduction. - L'écoulement laminaire en convection naturelle entre deux plaques planes verticales et parallèles a déjà été étudié dans le cas de conditions de températures constantes aux parois, en régime établi [1] et pour la zone de longueur d'entrée [2]. D'autres travaux considèrent également le cas où les parois sont chauffées de façon asymétrique et les résultats sont donnés pour un écoulement développé [3] et pour la zone d'entrée [4]. Par contre, 
l'écoulement de convection mixte dans une configuration semblable n'a fait l'objet que de peu d'études, qui se sont en général limitées au cas où l'écoulement est développé, avec l'hypothèse d'une température de paroi variant linéairement [5].

Le problème de l'écoulement développé en convection mixte dans un canal plan vertical dont les parois sont chauffées à flux constant a été abordé principalement de manière expérimentale [6], mais le problème théorique n'a pas été résolu dans la mesure où la température du fluide à la paroi était supposée connue et n'était donc pas déduite des équations. L'objet de cette étude est d'obtenir les solutions générales de l'écoulement de convection mixte laminaire dans un canal vertical bidimensionnel dont les parois sont soumises à des densités de flux thermique constantes mais non nécessairement égales.

Un tel écoulement existe en particulier dans les collecteurs plans, dans lesquels le flux solaire crée un courant d'air chaud ascendant par convection.

Nous présentons successivement les résultats concernant le régime établi de l'écoulement, puis la zone de développement des couches limites thermiques et dynamiques. Enfin, des mesures effectuées sur un dispositif expérimental sont comparées aux résultats numériques.

2. Equations de la convection mixte dans le canal. 2.1 HYPOTHÈSES GÉNÉRALES. - Considérons un canal vertical bidimensionnel formé de deux plaques planes parallèles (Fig. 1). Le conduit est ouvert à ses extrémités supérieure et inférieure sur deux milieux semiinfinis au repos, caractérisés par une température et

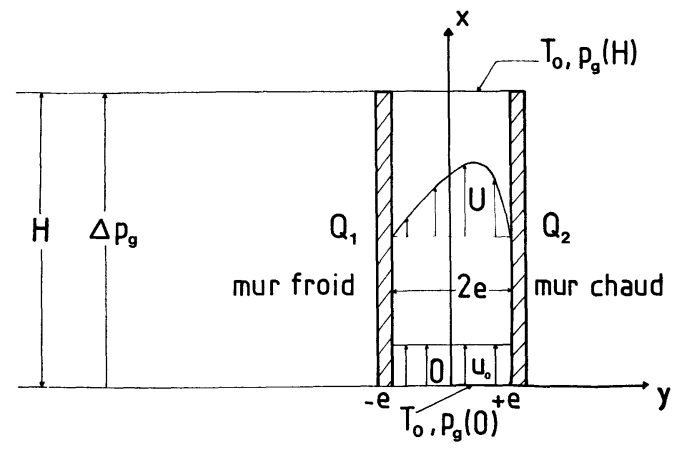

Fig. 1. - Collecteur plan à deux dimensions.

[Two dimensional flat plate collector.]

une pression motrice constantes. A l'entrée du conduit, la valeur de la pression motrice est déterminée en supposant qu'elle satisfait au théorème de Bernoulli. De même, la pression motrice dans la section de sortie est supposée vérifier la condition de jet en atmosphère libre, elle est donc égale à celle qui règne à l'extérieur. Le fluide pénètre dans le canal à la température ambiante $T_{0}$ et avec une vitesse uniforme $u_{0}$. L'écoulement du fluide dans le conduit, supposé laminaire et stationnaire, est régi par les lois de la convection mixte, et le fluide satisfait aux hypothèses de Boussinesq.

2.2 EquATIONS DimENSIONNELles. - Dans le cadre de l'approximation de la couche limite, les équations du mouvement, de l'énergie et de continuité s'écrivent sous forme dimensionnelle, en prenant pour température de référence la température ambiante $T_{0}$ :

$$
\left\{\begin{array}{l}
u \frac{\partial u}{\partial x}+v \frac{\partial u}{\partial y}=-\frac{1}{\rho} \cdot \frac{\mathrm{d} p_{\mathrm{g}}}{\mathrm{d} x}+\beta g\left(\theta_{\mathrm{d}}-T_{0}\right)+v \frac{\partial^{2} u}{\partial y^{2}} \\
u \frac{\partial \theta_{\mathrm{d}}}{\partial x}+v \frac{\partial \theta_{\mathrm{d}}}{\partial y}=a \frac{\partial^{2} \theta_{\mathrm{d}}}{\partial y^{2}} \\
\frac{\partial u}{\partial x}+\frac{\partial v}{\partial y}=0
\end{array}\right.
$$

où $p_{\mathrm{g}}$ est la pression motrice définie par :

$$
p_{\mathrm{g}}(x)=p(x)+\rho_{0} g x
$$

$p$ étant la pression régnant à l'abscisse $x$ dans le canal et $\rho_{0}$ la masse volumique du fluide à la température de référence $T_{0}$.

Sur les parois du canal, on écrit les conditions d'adhérence du fluide et les conditions de densités de flux thermique constantes, soit :

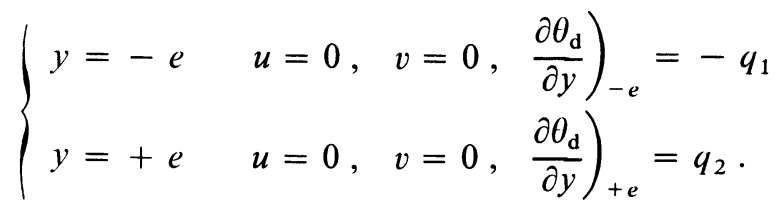


2.3 NOMBRES ADIMENSIONNELS. - Les solutions des éqs. (1), (2) et (3) dépendent de cinq paramètres adimensionnels qui sont :

- L'épaisseur réduite du canal :

$$
l=\frac{2 e}{H} .
$$

- Le nombre de Prandtl :

$$
\operatorname{Pr}=\frac{v}{a} .
$$

- Le nombre de Grashof modifié :

$$
G r_{\mathrm{e}}=\frac{\beta g e^{4}\left(q_{1}+q_{2}\right)}{v^{2} \lambda} .
$$

- Le rapport des flux sur les parois :

$$
Q=\frac{q_{2}-q_{1}}{q_{2}+q_{1}} .
$$

- Un paramètre mesurant l'influence de la convection forcée sur la convection naturelle :

où

$$
P=\frac{\lambda \Delta \rho_{\mathrm{g}}}{\operatorname{H\rho \beta ge}\left(q_{1}+q_{2}\right)}
$$

$$
\Delta p_{\mathrm{g}}=p_{\mathrm{g}}(H)-p_{\mathrm{g}}(0) .
$$

3. Ecoulement en régime établi. - 3.1 DÉfINITION DE L'ÉCOULEMENT DÉveLOPPÉ. - L'écoulement de régime établi en convection mixte est caractérisé par les relations :

$$
\left\{\begin{array}{l}
u(x, y)=u(y) \\
v(x, y)=0 \\
\theta_{\mathrm{d}}(x, y)=T_{\mathrm{md}}(x)+T_{\mathrm{d}}(y)
\end{array}\right.
$$

où $T_{\mathrm{d}}(y)$ représente le profil de température dans une section du canal et $T_{\mathrm{md}}(x)$, la température moyenne définie par :

$$
T_{\mathrm{md}}(x)=\frac{\int_{-e}^{+e} \rho u \theta_{\mathrm{d}} \mathrm{d} y}{\int_{-e}^{+e} \rho u \mathrm{~d} y} .
$$

La température moyenne $T_{\text {md }}$ est une fonction linéaire de l'abscisse $x$ dans le canal et s'écrit :

$$
T_{\mathrm{md}}(x)=A x+T_{0}
$$

où $A$ représente le gradient de température moyenne et est constant.

3.2 Equations adimensionNelles. - Quand l'écoulement est pleinement développé, on définit les variables adimensionnelles suivantes :

$$
\left.\begin{array}{rl}
X & =\frac{x}{e}, \quad Y=\frac{y}{e}, \quad U=\frac{u e}{v}, \quad V=\frac{v e}{v} \\
T & =\frac{\lambda T_{\mathrm{d}}}{\left(q_{1}+q_{2}\right) e}, \quad T_{\mathrm{m}}=\frac{\lambda\left(T_{\mathrm{md}}-T_{0}\right)}{\left(q_{1}+q_{2}\right) e} \\
\theta & =\frac{\lambda \theta_{\mathrm{d}}}{\left(q_{1}+q_{2}\right) e} \\
P_{\mathrm{g}} & =\frac{p_{\mathrm{g}}}{\rho(v / e)^{2}}
\end{array}\right\}
$$

et les relations (12), (13) et (14) s'écrivent :

avec

$$
\left\{\begin{array}{l}
U(X, Y)=U(Y) \\
V(X, Y)=0 \\
0(X, Y)=T(Y)+T_{\mathrm{m}}(X)
\end{array}\right.
$$

où $t$ est un paramètre défini par :

$$
t=\left(\frac{A \beta g e^{4}}{v a}\right)^{1 / 4} .
$$

Dans ces conditions, le système d'éq. (1), (2) et (3) se réduit à :

$$
\left\{\begin{array}{l}
\frac{\mathrm{d}^{4} T(Y)}{\mathrm{d} Y^{4}}+t^{4} \cdot T(Y)+t^{4}\left(\frac{t^{4}}{G}-P\right)=0 \\
U(Y)=\frac{G r_{e}}{t^{4}} \cdot \frac{\mathrm{d}^{2} T(Y)}{\mathrm{d} Y^{2}}
\end{array}\right.
$$

où $G$ est un nombre de Rayleigh défini par :

$$
G=l . P r . G r_{\mathrm{e}} .
$$

De même, les conditions aux limites (5) s'écrivent :

$$
\left.\begin{array}{l}
U(+1)=0 \\
U(-1)=0 \\
\left.\frac{\mathrm{d} T}{\mathrm{~d} Y}\right)_{-1}=\frac{Q-1}{2} \\
\left.\frac{\mathrm{d} T}{\mathrm{~d} Y}\right)_{+1}=\frac{Q+1}{2}
\end{array}\right\}
$$

De plus on a :

$$
\int_{-1}^{+1} T(Y) \cdot \frac{\mathrm{d}^{2} T(Y)}{\mathrm{d} Y^{2}} \cdot \mathrm{d} Y=0
$$

qui se déduit directement de (15).

Un paramètre important est le gradient adimensionnel de température moyenne $t$. Pour une valeur donnée et arbitraire de ce paramètre, $S$. Ostrach [7] a calculé les solutions analytiques des éq. (23) et (24). Mais en général, la valeur de ce paramètre est inconnue à priori, et un processus itératif doit être utilisé pour résoudre ces équations. 
3.3 Résultats. - L'importance relative de la convection forcée et de la convection naturelle dépend à priori de la valeur des paramètres $P, G$ et $Q$. Les profils de vitesse et de température obtenus dans le cas de l'air $(P r=0,71)$ montrent (Fig. 2) que la valeur de $P$ détermine l'influence de la convection forcée sur la
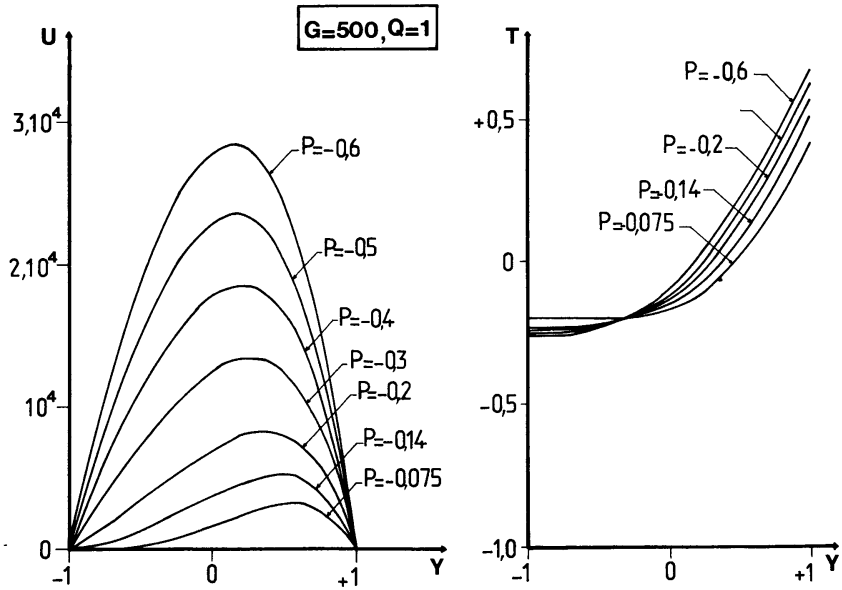

Fig. 2. - Profils adimensionnels de vitesse et de température transversale.

[Dimensionless velocity and transverse temperature profiles.]

convection naturelle. Pour des valeurs absolues de $P$ faibles $(|P|<0,15)$, la convection naturelle est prédominante et les profils de vitesse se rapprochent des profils de couche limite. Dans le domaine où $0,15<|P|<0,3$, l'écoulement est nettement de convection mixte. Pour des valeurs absolues de $P$ grandes $(|P|>0,3)$, la convection forcée prédomine et les profils de vitesse tendent vers les profils obtenus pour l'écoulement de Poiseuille.

4. Zone de développement des couches limites. L'écoulement n'est pleinement développé qu'à partir d'une certaine hauteur dans le canal, et une zone d'entrée de longueur non négligeable doit être considérée.

4.1 Equations admensionnelles. - Dans cette zone, on définit les variables adimensionnelles suivantes :

$$
\left.\begin{array}{rlrl}
Y & =\frac{y}{e}, & V & =\frac{v e}{v} \\
X^{\prime} & =\frac{x}{H G r}, & U^{\prime} & =\frac{e^{2} u}{H v G r} \\
\theta^{\prime} & =\frac{\left(\theta_{\mathrm{d}}-T_{0}\right) \lambda}{e\left(q_{1}+q_{2}\right)}, & P_{\mathrm{g}}^{\prime} & =\frac{p_{\mathrm{g}} e^{4}}{\rho H^{2} v^{2} G r^{2}}
\end{array}\right\}
$$

où $G r$ est un nombre de Grashof modifié défini par :

$$
G r=\frac{l}{2} \cdot G r_{\mathrm{e}} .
$$

Dans ces conditions, les éqs. (1), (2) et (3) dans la zone d'entrée s'écrivent sous forme adimensionnelle :

$$
\left\{\begin{array}{l}
U^{\prime} \frac{\partial U^{\prime}}{\partial X^{\prime}}+V \frac{\partial U^{\prime}}{\partial Y}=-\frac{\mathrm{d} P_{\mathrm{g}}^{\prime}}{\mathrm{d} X^{\prime}}+\theta^{\prime}+\frac{\partial^{2} U^{\prime}}{\partial Y^{2}} \\
U \frac{\partial \theta^{\prime}}{\partial X^{\prime}}+V \frac{\partial \theta^{\prime}}{\partial Y}=\frac{1}{P r} \cdot \frac{\partial^{2} \theta^{\prime}}{\partial Y^{2}} \\
\frac{\partial U^{\prime}}{\partial X^{\prime}}+\frac{\partial V}{\partial Y}=0 \\
M=\frac{1}{2} \int_{-1}^{+1} U^{\prime} \mathrm{d} Y
\end{array}\right.
$$

où $M$ est le débit adimensionnel dans la conduite. La différence de pression motrice adimensionnelle $D P_{\mathrm{g}}$, donnée, s'ajoute à la pression motrice sans dimension à l'entrée du canal. Les conditions aux limites associées aux éqs. (29), (30), (31) et (32) peuvent alors s'écrire :

$$
\begin{aligned}
& \text { Pour } \quad X^{\prime}=0 \text { et } \quad-1<Y<+1: U^{\prime}=M, \quad V=0, \quad \theta^{\prime}=0 . \\
& \text { Pour } \left.\quad X^{\prime} \geqslant 0 \quad \text { et } \quad Y=-1 \quad: U^{\prime}=0, \quad V=0, \quad \frac{\partial \theta^{\prime}}{\partial Y}\right)_{-1}=\frac{Q-1}{2} . \\
& \text { Pour } \left.X^{\prime} \geqslant 0 \text { et } Y=+1 \quad: U^{\prime}=0, \quad V=0, \frac{\partial \theta^{\prime}}{\partial Y}\right)_{+1}=\frac{Q+1}{2} . \\
& \text { Pour } \quad X^{\prime}=0 \quad: P_{\mathrm{g}}^{\prime}=-\frac{1}{2} \cdot M^{2}+D P_{\mathrm{g}} \text {. } \\
& \text { Pour } \left.\quad X^{\prime}=L \quad \text { (correspondant à } x=H\right): P_{\mathrm{g}}^{\prime}=0 \text {. }
\end{aligned}
$$

D'après la définition de $X^{\prime}$, la hauteur sans dimension $L$ du canal est l'inverse du nombre de Grashof $G r$. Les éqs. (29), (30), (31) et (32) sont résolues à l'aide d'une méthode numérique aux différences finies voisine de celle utilisée par Bodoia et Osterle [2]. Le système d'éqs. (29), (30), (31) et (32) et ses conditions aux limites (33) dépend de trois paramètres $\operatorname{Pr}, Q$ et $M$. Les champs de vitesse, pression et tempé- 
rature dans toute la conduite sont calculés par une procédure pas à pas depuis l'entrée du canal jusqu'à l'abscisse $X^{\prime}=L$ pour laquelle $P_{\mathrm{g}}^{\prime}=0$.

4.2 Résultats. - Dans le cas du chauffage asymétrique des parois $(Q=1)$, et si le fluide est de l'air $(\operatorname{Pr}=0,71)$, les courbes de la figure 3 indiquent les valeurs du débit $M$ en fonction de $L$, en convection naturelle et pour un exemple de convection mixte. Il est à remarquer que la valeur de $M$ ne tend pas vers une limite finie pour les grandes valeurs de $L$, contrairement à ce qui est obtenu dans le cas où les températures des parois de la conduite sont maintenues constantes [2].

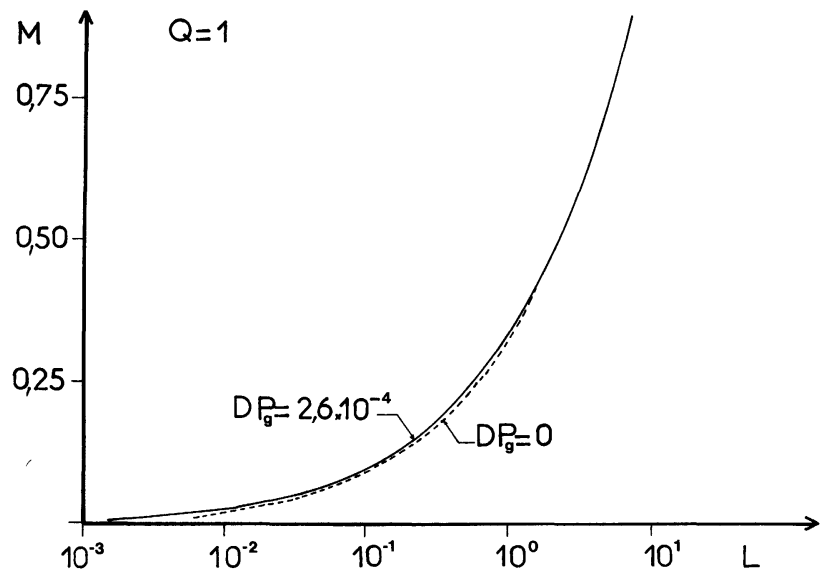

Fig. 3. - Débit massique en fonction de la hauteur du collecteur pour la convection naturelle et la convection mixte.

[Flow rate versus channel length for free and mixed convection.]

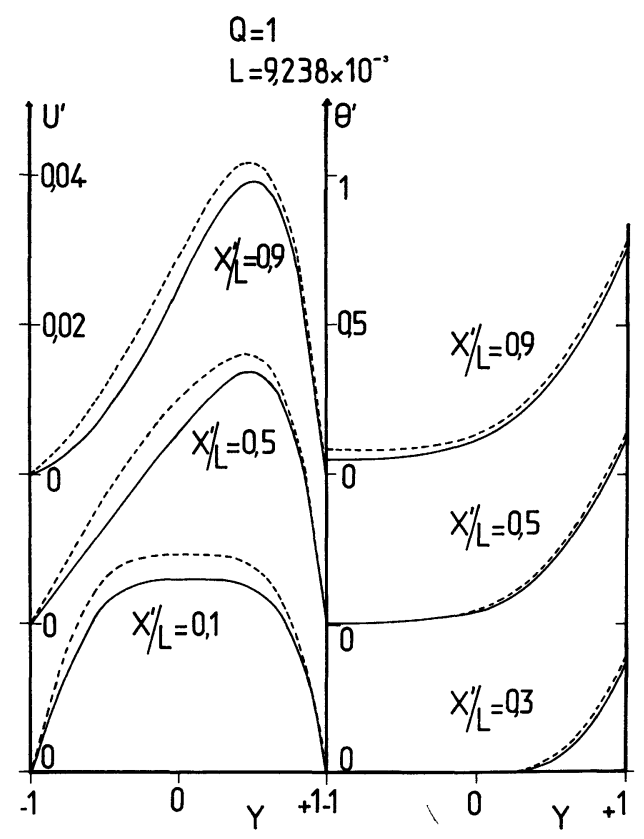

Fig. 4. - Profils de vitesse et de température en convection naturelle (ligne continue) et en convection mixte (ligne pointillée).

[Velocity and temperature fields in the case of free convection (continuous line) and in the case of mixed convection (stippled line).]
Pour une valeur de $L=9,238 \times 10^{-3}$, les profils de vitesse et de température en différentes sections du canal sont représentés figure 4 , pour un chauffage asymétrique des parois $(Q=1)$, en convection naturelle et dans un cas de convection mixte $\left(D P_{\mathrm{g}}=0,26 \times 10^{-3}\right)$. Il apparaît que les profils de température obtenus en convection naturelle et en convection mixte sont assez voisins. La figure 4 montre également que le profil des vitesses évolue d'une vitesse d'entrée uniforme vers un profil de couche limite quand l'abscisse dans la conduite augmente. La dissymétrie de l'écoulement est atténuée dans le cas de la convection mixte.

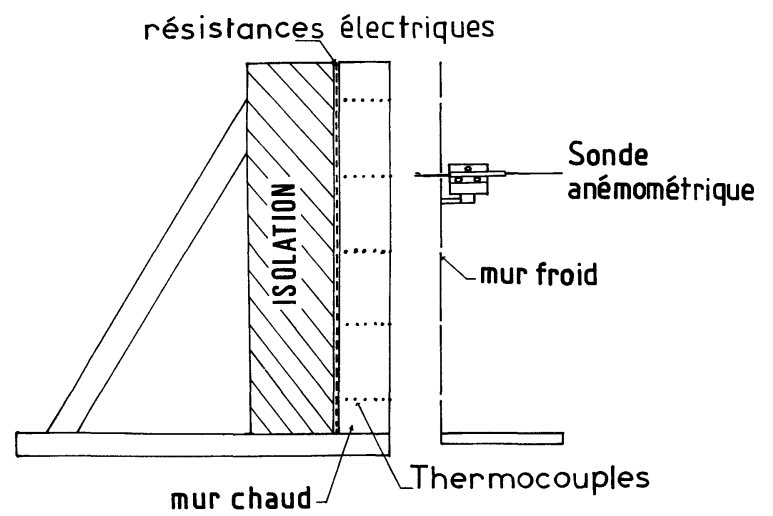

Fig. 5. - Dispositif expérimental.

[Experimental device.]

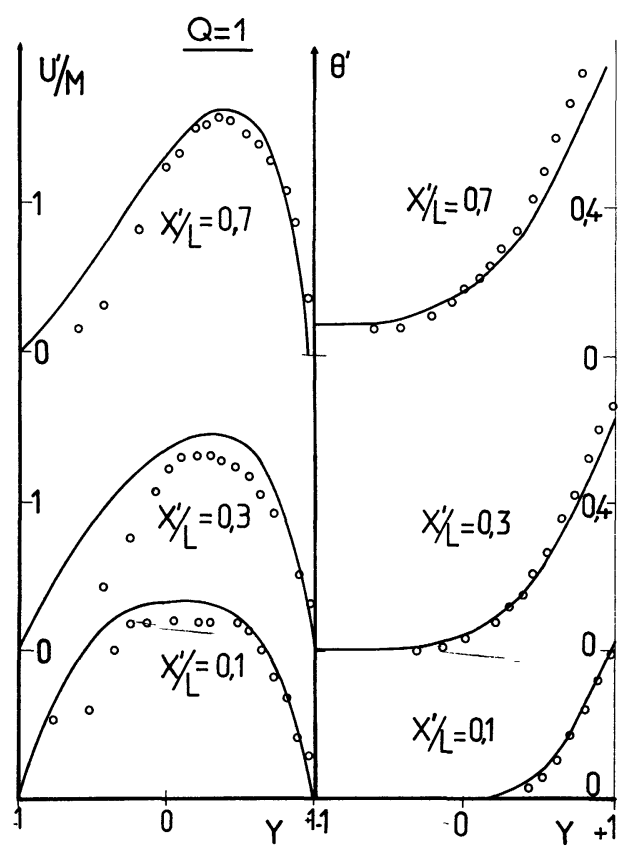

Fig. 6. - Comparaison entre les données expérimentales (points) et les profils de vitesse et de température calculés (ligne continue).

[Comparison between experimental data (points) and computed velocity and temperature profiles (continuous line).] 
5. Mesures expérimentales. - 5.1 DispositiF EXPÉRIMENTAL. - Dans le but de vérifier la validité des résultats numériques, un conduit dont on peut faire varier l'épaisseur a été réalisé (Fig. 5). Une des faces du canal est composée d'un mur en béton de $2,5 \mathrm{~m}$ de haut et de $1,0 \mathrm{~m}$ de large, chauffé par des résistances électriques et isolé thermiquement sur sa partie externe. L'autre face du conduit est constituée d'un matériau réfléchissant le rayonnement infrarouge. Les vitesses et les températures du fluide dans le canal sont mesurées avec une sonde anémométrique à fil chaud.

5.2 Résultats. - Des mesures ont montré que pour un rapport $2 e / H<0,06$, l'existence d'un écoulement laminaire est déterminée par la valeur de l'épaisseur du canal, et donc par un nombre de Reynolds, plus que par un nombre de Grashof défini à partir de l'abscisse sur les plaques. Ainsi la transition entre les écoulements laminaire et turbulent a été mise en évidence quand l'épaisseur du conduit est de l'ordre de $3 \mathrm{~cm}$ pour les densités de flux pariétales utilisées (environ $100 \mathrm{~W} / \mathrm{m}^{2}$ ).

Dans le cas où le canal a une épaisseur de $2 \mathrm{~cm}$, l'écoulement est laminaire. On compare alors (Fig. 6) les profils de vitesse et de température mesurés à différentes hauteurs dans la conduite aux profils calculés numériquement. Ces différents résultats sont en bon accord, compte tenu d'une part de la difficulté à contrôler le décollement des couches limites à l'entrée, et d'autre part des imprécisions introduites par le dispositif de mesure aux faibles vitesses.

En dépit de ces quelques difficultés, ces expériences montrent que les calculs numériques effectués dans le cas d'un canal à flux constant sont représentatifs de l'écoulement dans un collecteur plan réel.

\section{Bibliographie}

[1] OSTRACH, S., Laminar natural convection flow and heat transfer of fuids in channels with constant wall temperature. NACA TN 2863 (1952)

[2] Bodoia, J. R. and Osterle, J. F., The development of free convection between heated vertical plates. J. Heat Transfer 84 (1962) 40.

[3] Aung, W., Fully developed laminar free convection between vertical plates heated asymmetrically. Int. J. Heat Mass Transfer 15 (1972) 1577.

[4] Aung, W., Fletcher, L. S., Sernas, V., Developing laminar free convection between vertical flat plates with asymmetric heating. Int. J. Heat Mass Transfer 15 (1972) 2293.
[5] Ostrach, S., Combined natural and forced convection laminar flow and heat transfer of fluids with and without heat sources in channels with linearly varying wall temperatures. NACA TN 3141 (1954).

[6] LAUBer, T. S. and WelCH, A. U., Natural convection heat transfer between vertical flat plates with uniform heat flux. Proc. of third Int. Heat Transf. Conf. Chicago 126 (1966).

[7] Ostrach, S., Internal viscous flows with body forces, Boundary layer Research (Symposium Freiburg) 185 (1958). 